\title{
Thoracic migration of silicone gel after breast implant rupture: a case report and literature review
}

\author{
Benedetta Fanelli ${ }^{1}$. Marco Marcasciano ${ }^{2}$ - Stefano Lovero ${ }^{1,3} \cdot$ Luca Codolini $^{1} \cdot$ Donato Casella $^{2}$. \\ Maria Giuseppina Onesti ${ }^{1} \cdot$ Diego Ribuffo $^{1} \cdot$ Nicolo Scuderi $^{1}$
}

Received: 7 December 2020 / Accepted: 2 February 2021 / Published online: 17 February 2021

(C) The Author(s) 2021

\begin{abstract}
Nowadays silicone is a widespread material for medical devices. In particular, it is commonly used for implants manufacturing, for that patients undergoing breast augmentation or breast reconstruction after mastectomy. However, the use of silicone implants is not free from risks. Ruptures of silicone breast implants are uncommon, in general post-traumatic or iatrogenic, and usually related to implant's wall weakness of unknown origin but probably due to biochemical reactions that cause wall rupture. As a consequence of a rupture, silicone gel from damaged implants may have a continuity migration to the chest wall, axillae, and upper extremities, resulting in granulomatous inflammation or siliconoma, or a lymphatic migration to axillary lymph nodes. In this regard, silicone thoracic migration is extremely rare, and nowadays a leakage is unlikely to happen with more modern cohesive silicone gel implants. Nevertheless, procedures such as thoracic surgery and thoracotomies may be responsible for accidental breast implant rupture, capsular discontinuity, and eventually intrathoracic silicone migration, especially when dealing with older generations of breast implants. We report a rare case of a 75 -year-old woman presenting with pleural silicone effusion, 18 years after a right breast reconstruction for breast cancer, followed by right upper lobe resection for a lung carcinoma. A combination of muscular flap and DTI pre-pectoral breast reconstruction with biological membrane (ADM) has been used for treatment. Literature was reviewed for cases of breast implants free silicone localization in the chest cavity, focusing on previous surgeries, anamnestic relevances, and surgical management.

Level of Evidence: Level V, risk/prognostic study.
\end{abstract}

Keywords Silicone breast implant $\cdot$ Breast surgery $\cdot$ Reconstructive surgery $\cdot$ Silicone complications

\section{Introduction}

Nowadays silicone is a widespread material for medical devices. In particular, it is commonly used for implants manufacturing, for that patients undergoing breast augmentation or breast reconstruction after mastectomy. More than 1.5

Stefano Lovero

stefano.lovero@uniroma1.it

1 Department of Plastic and Reconstrutive Surgery, School of Medicine, University of Rome "La Sapienza", Policlinico Umberto I, Viale del Policlinico, 155 Roma, Italy

2 Unità di Oncologia Chirurgica Ricostruttiva della Mammella, "Spedali Riuniti" di Livorno, Breast Unit Integrata di Livorno, Livorno, Italia

3 Sapienza University of Rome, Piazzale Aldo Moro, 5, 00185 Roma, RM, Italy million women in the USA have silicone breast implants [1]. In Europe, about two million women have undergone heterologous breast augmentation, while hundreds of thousands have experienced breast reconstruction surgery with silicone implants [2]. The widespread application of silicone implants is a consequence of its biological stability and physical properties, combined with minimal tissue reaction and lack of immunogenicity [3]. However, the use of silicone implants is not free from risks. Most common complications related to the application of silicone gel filled breast implants include capsular contracture, rupture, wrinkling, asymmetry, scarring, pain, exposure, and infection. Rare rupture of silicone breast implants is usually post-traumatic or iatrogenic, usually related to implant's wall weakness of unknown origin but probably due to biochemical reactions that cause wall rupture [4]. As a consequence of a rupture, silicone gel from damaged implants can have a continuity migration to the chest wall, axillae, and upper extremities, resulting in granulomatous inflammation or 
siliconomas or a lymphatic migration to axillary lymph nodes. In this regard, silicone thoracic migration is extremely rare. Surgical procedures as thoracic surgery and thoracotomy may cause a breast implant rupture and capsular discontinuity and may be related to intrathoracic silicone migrations, above all when dealing with older breast implant generations. The first case of pleural effusion caused by the rupture of a silicone mammary prosthesis was reported by Stevens et al. in 1987 [5]. Since then, pleural silicone granulomas have rarely been reported in literature [6]. We report on a rare case of a 75-yearold woman presenting with pleural silicone effusion, 18 years after a right breast reconstruction for breast cancer. Extracapsular implant rupture was diagnosed, putting in evidence an exceptional silicone gel fistulization into the pleural space, through a thoracic defect. A technique with a combined muscular flap and pre-pectoral breast reconstruction with biological membrane has been used for treatment. Literature was reviewed for cases of breast implants free silicone localization in the chest cavity, focusing on previous surgeries, anamnestic relevances, and surgical management.

\section{Case report}

In May 2018, a 75-year-old woman presented at our Breast Unit Department for right breast deformity (Fig. 1 and Fig. 2). She had medical history of treated hypertension, smoking (15 cigarettes/die), gastroesophageal reflux, and serological positivity for $\mathrm{HCV}$. Patient referred a previous right mastectomy and ipsilateral axillary lymphadenectomy for a ductal infiltrating breast cancer, followed by hormone therapy and radiotherapy (February 1990). Delayed retro-pectoral reconstruction with definitive round smooth breast implant (Sebbin, $515 \mathrm{cc}$ ) and contralateral breast reduction was performed in December 1990. In June 2015, she had

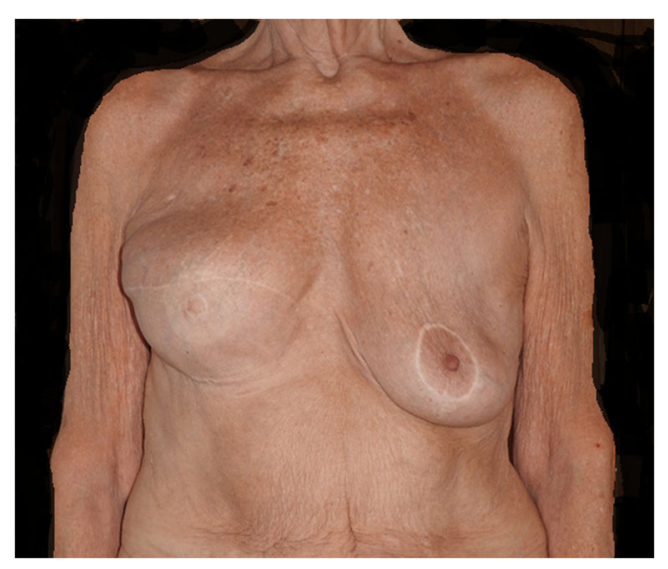

Fig. 1 Preoperative right breast deformity undergone a right upper lobe resection for a lung carcinoma. Total body CT scan was performed in another institution: results showed intra-capsular prosthetic rupture in the right side as well as an extra-capsular rupture in the inferior-medial pole with intercostal prosthetic gel effusion and compression of lung parenchyma (Fig. 3). A breast MRI with contrast was carried out. Images showed the presence of a sub-pectoral breast implant with signs of intra- and extra-capsular rupture in medial pole. A prosthetic intrathoracic gel effusion through a thoracic wall defect of $35 \mathrm{~mm}$ was detected. A right upper lung lobe compression was evident at the level of III and IV rib. The herniated fluid was $30 \times 60$ $\mathrm{mm}$. The silicone diffusion into the chest cavity was suspected to be the consequence of the right upper lung resection causing iatrogenic communication between the breast implant pocket and the chest cavity. Full communication and virtuous cooperation among plastic surgeons, radiologists, and cardio-thoracic surgeons have been essential for confirming the diagnostic hypothesis as well as for targeting a virtuous multidisciplinary management of the case.

Surgical implant removal was planned after multidisciplinary consultation. Before surgery, patient's routine laboratory test was within normal limits. Surgical access was performed through previous equatorial scar. An irregular peri-prosthetic capsule was soon identified during right breast dissection. Opening the peri-prosthetic capsule, silicone gel was drained out of the breast cavity. The implant resulted destroyed with wide silicone leaking, and only few fragments of prosthetic membrane were identifiable. Interestingly, capsule was very adherent to the surrounding tissues (pectoralis major muscle band, subcutaneous fat, thoracic wall, ribs). A complex capsulectomy was performed until thoracic breach. Similar mixed fluid with silicone gel was found down in the thoracic cavity and was gently removed

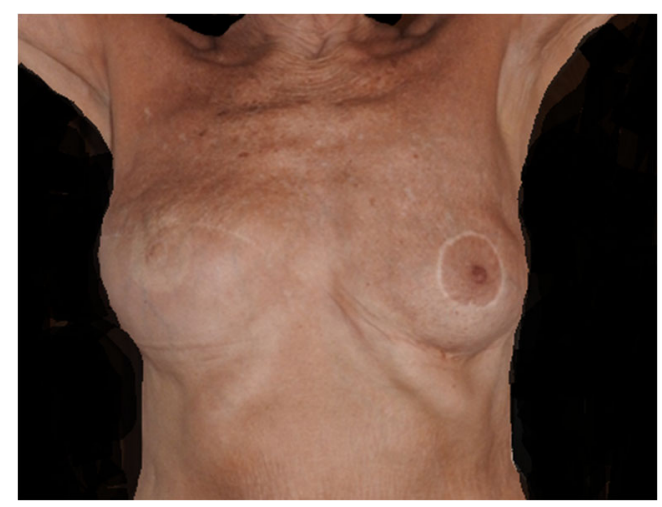

Fig. 2 Preoperative right breast deformity 
with a mechanical cleaning of the cavity. Functional tests of lung iper- and ipo-expansion were performed. It was not necessary to dissect pleural cavity. An accurate hemostasis and washing with saline solution were performed. Thoracic wall reconstruction was performed using a pectoralis major flap sutured in vicryl $3 / 0$. Suction drainage was placed into the pocket. We performed a pre-pectoral breast reconstruction with round smooth prosthetic (Mentor, $300 \mathrm{cc}$ ) and biological membrane (Braxon), fixed to the thoracic wall with stitches in vicryl 2/0. Suction drainage and aesthetic suture were placed. A contralateral breast symmetrization with a prepectoral round smooth prosthetic (Mentor, $150 \mathrm{cc}$ ) was performed at the same time. No complications were reported during the immediate postoperative period. The postoperative course was uneventful, and the patient was discharged on the 4 th postoperative day. At 3and 6-month follow-up, no complications occurred (Video: Clinical Case and Surgical Treatment).

\section{Literature review}

In September 2018, we performed a search in PubMed (National Library of Medicine, NLM) with the aim of finding similar cases in the literature. We used the PubMed keywords shown below: breast OR/AND (implant OR prosthesis) AND (rupture OR effusion) AND/OR (silicon OR siliconoma) AND (thorax OR thoracic OR intrathoracic OR pleura OR pleural). The searches were conducted with no date or language limits. The databases were searched from the inception date forward. The reference lists of the selected papers were subsequently reviewed for additional papers. Full-text papers were retrieved according to the selected abstracts and read and screened by three authors. We included all studies describing intra-thoracic complications of previous breast implant surgery. All studies were case reports. The included studies were

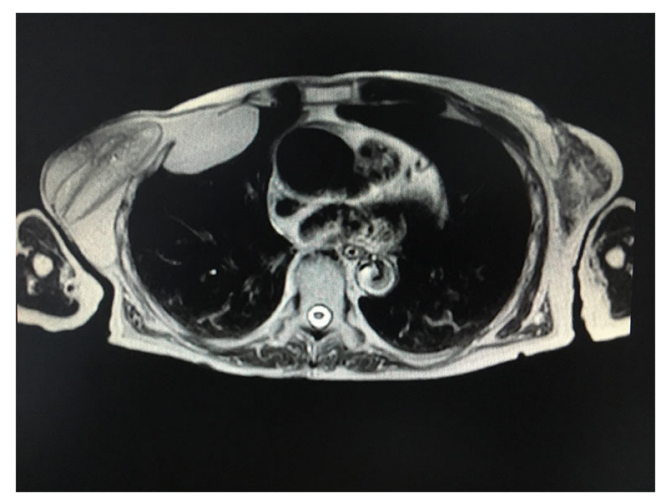

Fig. 3 Silicone effusion in total body CT reviewed using a descriptive checklist including the first author, year of publication, patient characteristic, prosthesis characteristic, and other surgeries than that of prosthesis implantation and anamnestic relevances (Table 1). We retrieved 17 articles that were all reviewed as described above. The included papers were published from 1987 to 2015.

We retrieved 40 articles, which was reduced to 26 after removing duplicates. These 26 articles were reviewed as described above, only 17 papers that described implants ruptures with silicone thoracic migration were described and clearly defined. We found several studies describing different silicone diffusion or suspected silicone polmonitis that were not included.

\section{Discussion}

Silicone has been used for many years in cosmetic and reconstructive breast surgery. When first introduced, it was thought to be relatively inert and to cause minimal tissue reaction. However, there have been several reports of foreign body reactions occurring in response to the presence of free silicone, both in animal experiments' and in clinical practice [7-12]. The histological features reported in these cases have shown mixed cellular infiltrate of histiocytes, lymphocytes, eosinophils, multinuclear giant cell granulomas, and macrophages with foamy cytoplasm. As in the case presented, after silicone implant rupture, free silicone may migrate throw areas of least resistance. The frequency of rupture of silicone breast implants is unknown but is a well-known occurrence, occurring at the time of insertion or during manual compression, after chest wall trauma or by iatrogenic causes. A minimum of $15 \%$ of modern implants can be expected to rupture between the third and tenth year after implantation [13]. Brown et al. found that $77 \%$ of women with silicone breast implants, without regard to complaints or symptoms, had at least one breast implant rupture; median implant age at the time of rupture was 10.8 years [14]. Extruded silicone, due to a breast implant failure, causes areas of inflammation, silicone granulomas, in the breast and surrounding tissues, including axillary lymph nodes, leading to the formation of pseudotumors [15, 16].Diagnosis of ruptured implants is difficult and is performed with physical examination, mammography, breast ultrasound, CT, and MRI. None of these techniques can detect all ruptures; CT and MRI detect approximately $80 \%$ and $90 \%$, respectively $[17,18]$.

M.R. Stevens was the first author to report a pleural effusion occurring as a result of ruptured breast prostheses 5 years after implantation [5]. The implant rupture was caused by a chest trauma, and the author treated 


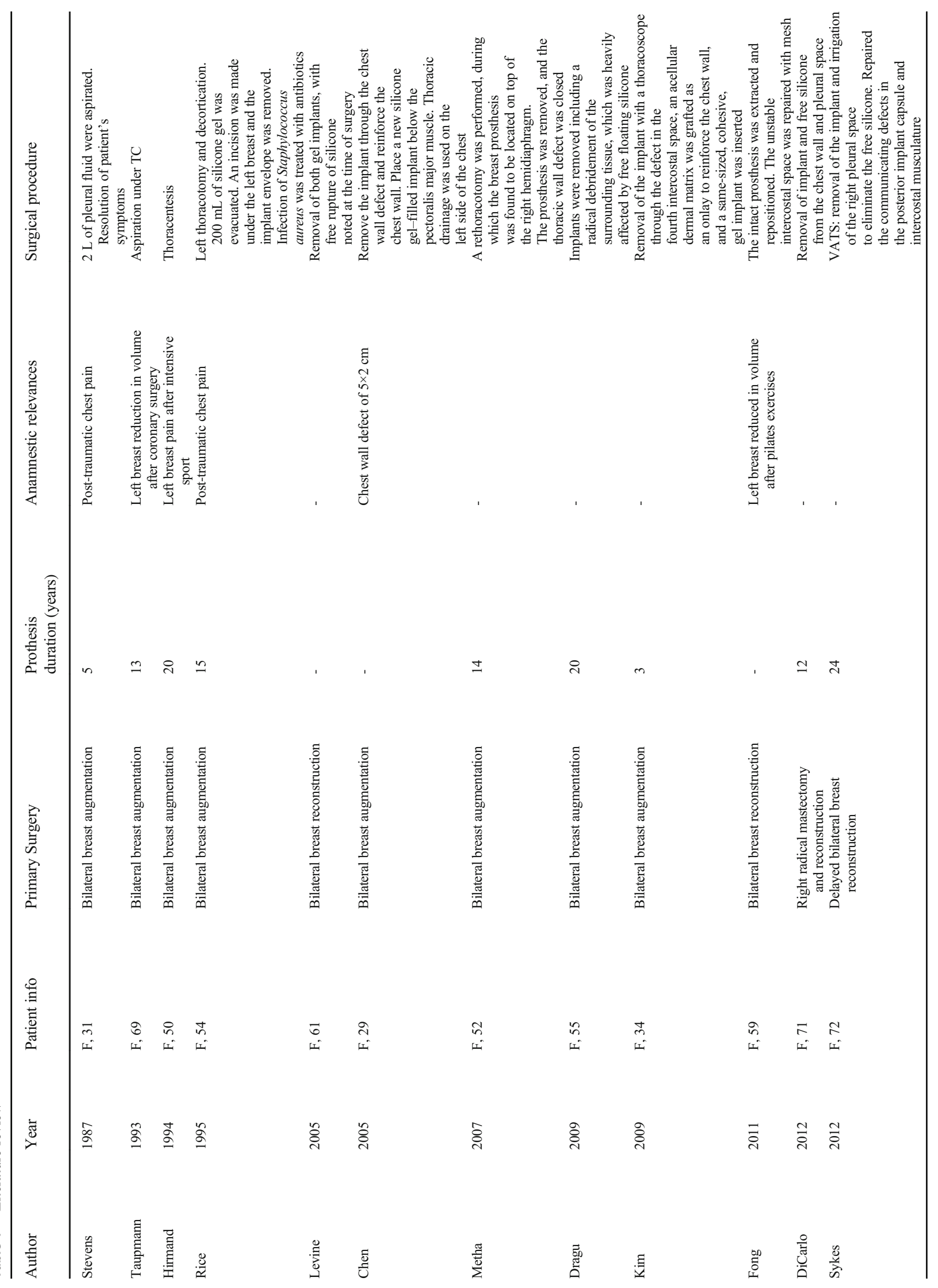




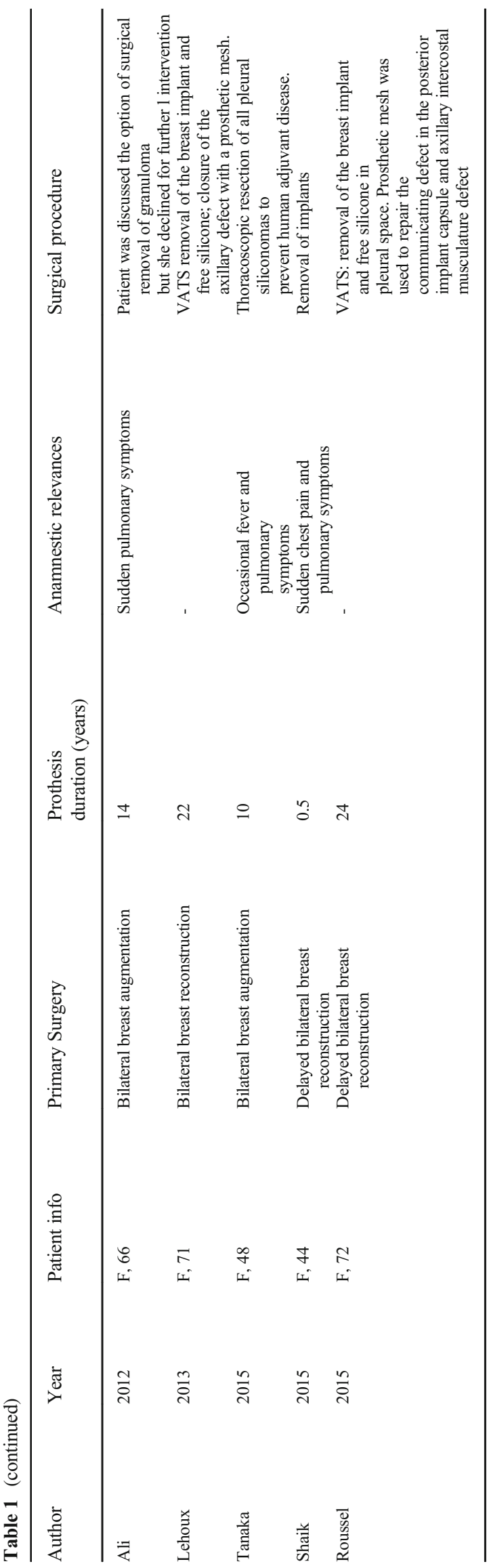

the pleural effusion with a complete aspiration of pleural fluid obtaining complete resolution of the symptoms. Even Hirdmand et al., in 1994, treated a similar pleural effusion by aspiration through a thoracentesis defect [19]. In this case, the implants were 20 years old (breast augmentation), and no clear etiology of migration was found. The patient only had capsulotomies 2 years after breast augmentation for capsular contracture. Breast implant failure associated to surgical procedures had instead been well documented in 1993, by Taupmann et al., that showed a singular case of silicone migration in pleural cavity due to a failed thoracocentesis under ultrasonic guidance. The breast prosthesis of a 13-yearold was nicked or traversed by the chest tube, allowing egress of silicone down the outside of the chest tube into pleural cavity [20]. Rice et al. in 1995 reported on a post-surgical silicone pleural effusion due to a complication of tube thoracostomy in the presence of mammary implants positioned 15 years before [21]. A tube thoracostomy was placed to drain a post-traumatic pleural effusion. Failure of this procedure nicked prosthesis integrity and developed a pleural effusion complicated by a Staphylococcus aureus supra-infection. Clinical resolution was obtained by thoracotomy and pleural decortication, silicone evacuation, breast implant removal, and antibiotics [22]. In 2005, Levine et al. and in 2015 Tanaka et al. described cases of fibrothorax, mimicking a mesothelioma, related to a breast implant rupture and silicone effusion during a cardiac-thoracic surgery procedure [23, 24]. Moreover, in 2009, Dragu et al. and in 2012 Di Carlo et al. reported a similar intrapulmonary silicon effusion, for aesthetic and reconstructive breast implant failure, respectively, related to lobectomy procedure and remote thoracic scar, mimicking breast metastasis or primitives lung cancer $[6,25]$. A similar case was reported by Sykes in 2012 and Lehoux in 2013, but silicone effusion occurred through a remote scar of chest wall caused by a video-assisted thoracoscopic surgery (VATS) procedure [26, 27]. Silicone effusion is not the only complication that may involve the chest cavity. In fact, a different pressure between intrathoracic cavity and extrathoracic tissues can lead to the complete migration of a breast implant through a chest wall interruption, elicited by physical exercise, compression, and implant liquidity [28-30]. Chest wall interruption may have different etiologies like trauma, congenital conditions and iatrogenic, as report by Metha et al. [31]. The authors describe a breast implant migration, 14 years later a breast augmentation, through a chest wall interruption due to thoracotomy for lung cancer. Another case of chest wall interruption after surgery was described in 2015 by Russel et al that shown an intrathoracic removal of breast implant following VATS procedure 
[32]. Silicone implant failure may be clinically silent, and usually, it is more likely to be found in the "old generation" silicone prosthesis placed more than two decades ago. As biomaterial science has recently developed, thanks to multilayers implants and cohesive silicone gel implants, the risk of implant failure and free silicone diffusion is greatly reduced. In fact, a leakage is unlikely to happen with more modern cohesive silicone gel implants, so this kind of complication is likely to become even more rare. Apart from some exceptional circumstances, in fact, cohesive silicone will not easily spread to the surrounding tissue; however, silicone present in older generations of implants can be subject to wide dissemination. For these reasons, some authors suggest replacing all implants used before the era of double lumen and cohesive silicone gel implants with new and modern implants [33, 34].We present a rare case of pleural silicone effusion through a chest wall defect with consequent granulomas, 18 years after a right breast reconstruction for breast cancer, treated with a multidisciplinary approach with thoracic surgeons and radiologists. We performed a breast implant removal, and fluid silicone was drained out of the breast cavity. Chest wall integrity was restored with a pectoralis major flap and a pre-pectoral breast reconstruction with the support of a biological membrane. To date, biomaterials evolution and surgical techniques evolution allow more accurate surgical treatments using breast implants with synthetic mesh and membrane [35-38]. In our case, we performed a pre-pectoral breast reconstruction with breast implant and synthetic membrane, using pectoralis major muscle to cover thoracic gap and restore chest wall integrity and to allow implant placement. Reviewing the literature, thoracic effusion of silicon after breast implant rupture is a rare event. Nevertheless, in the majority of cases recorded, a previous surgical procedure involving chest wall is reported. This condition may cause the interruption in the chest wall as well as develop a "locus minoris resistencia" at the level of the scars, representing prerequisites for a silicon migration in cases of extracapsular implant ruptures. We are glad to present an original surgical procedure to treat, for the first time, thoracic silicone effusion after breast implant rupture with a prepectoral major reconstruction using breast implant and synthetic membrane. Chest wall suture and pectoralis major flap have guaranteed restoration of chest wall integrity by a hermetic fistula closure. Functional and cosmetic results gratified patient and surgeons. As reported in our case, the patient did not refer any respiratory symptoms. Nevertheless, thoracic cavity involvement may be either clinically silent or symptomatic, depending on the pulmonary enrollment. Lung compression or bronchial obstruction may determine respiratory distress syndrome or acute, and chronic, pneumonitis events. In this regard, even though this is a not very common event, it still represents a real potential complication that plastic surgeons should keep in mind when dealing with patients with breast implants who have experienced or are planning surgical thoracic procedures. No official guidelines for follow-up after breast prosthesis implantation are currently available, though targeted radiological protocols should be considered in all patients with suspected breast implants rupture, who underwent surgical procedures involving the chest wall [33]. Cooperation among plastic surgeons, radiologists, and cardiothoracic surgeons should be mandatory in order to manage such a complex clinical scenario.

\section{Conclusions}

We presented a rare case of pleural migration of free silicone after right breast implants failure through a chest wall interruption. Literature has been deeply reviewed searching for all references about similar events. Chest wall surgical procedures and old generation breast implants represent to date the main risk factors of thoracic silicone migration, but the real incidence of these cases could be misdiagnosed or underestimated. Full communication among the different specialists involved in the multidisciplinary approach is always recommended.

Supplementary Information The online version contains supplementary material available at https://doi.org/10.1007/s00238-021-01799-0.

Funding Open Access funding provided by Università degli Studi di Roma La Sapienza.

\section{Declarations}

Ethics approval All procedures performed in studies involving human participants were in accordance with the ethical standards of the institutional and/or national research committee and with the 1964 Helsinki Declaration and its later amendments or comparable ethical standards. This is a case report and literature review. For this kind of studies no ethical approval is required.

Informed consent Informed consent was obtained from the patient.

Patient consent The patient signed informed consent regarding publishing her data and photographs. The patient has consented to the submission of the case report to the journal.

Conflict of interest B. Fanelli, M. Marcasciano, S. Lovero, L. Codolini, D. Casella, M.G. Onesti, Diego Ribuffo, and N. Scuderi declare that they have no conflict of interest.

Open Access This article is licensed under a Creative Commons Attribution 4.0 International License, which permits use, sharing, adaptation, distribution and reproduction in any medium or format, as long as 
you give appropriate credit to the original author(s) and the source, provide a link to the Creative Commons licence, and indicate if changes were made. The images or other third party material in this article are included in the article's Creative Commons licence, unless indicated otherwise in a credit line to the material. If material is not included in the article's Creative Commons licence and your intended use is not permitted by statutory regulation or exceeds the permitted use, you will need to obtain permission directly from the copyright holder. To view a copy of this licence, visit http://creativecommons.org/licenses/by/4.0/.

\section{References}

1. Bondurant S, Ernster V, Herdman R, ed. Safety of silicone breast implants. Committee on the Safety of Silicone Breast Implants, Division of Health Promotion and Disease Prevention, Institute of Medicine. Washington, DC: National Academy Press, 1999

2. Azavedo E, Boné B (1999) Imaging breasts with silicone implants. Eur Radiol 9(2):349-355

3. Ali L, Mcgivern D, Teoh R (2012) Rare disease: silicon granuloma mimicking lung cancer. BMJ Case Rep 2012:bcr2012006351

4. Fraldi M, Esposito L, Cutolo A, Carotenuto AR, Adamo C, Molea G (2016) Stealthy role of size-driven stresses in biomechanics of breast implants capsular contracture. J Mech Behav Biomed Mater 64:199-208

5. Stevens WMR, Burdon JGW, Niall JF (1987) Pleural effusion after rupture of silicone bag mammary prosthesis. Thorax. 42(10):825826

6. DiCarlo CM, Javadi P, Yang M, Gilkeson RC, Hsiao EM (2012) Pleural silicon granuloma mimics pleural metastasis: ruptured breast implant with silicone fistulising along a remote thoracotomy scar. Radiol Case Rep 7(3):672

7. Brody GL, Frey CF (1968) Peritoneal response to silicone fluid. Arch Surg 96:237-241

8. Vistnes LM, Bentley JW, Fogarty DC (1977) Experimental study of tissue response to ruptured gel-filled mammary protheses. Plast Reconstr Surg 59:31-34

9. Delage C, Shane JJ, Johnson FB (1973) Mammary silicone granuloma. Arch Dermatol 108:104-107

10. Wintsch W, Smahel J, Clodius L (1978) Local and regional lymph node response to ruptured gel-filled mammary protheses. Br J Plast Surg 31:349-352

11. Mason J, Apisarnthanarax P (1981) Migratory silicone granuloma. Arch Dermatol 117:366-367

12. Eisenberg HV, Bartels RJ (1977) Rupture of a silicone bag-gel breast implant by closed compression capsulotomy. Plast Reconstr Surg 59:849-850

13. Hölmich LR, Friis S, Fryzek JP, Vejborg IM, Conrad C, Sletting S, Kjøller K, McLaughlin JK, Olsen JH (Jul 2003) Incidence of silicone breast implant rupture. Arch Surg 138(7):801-806

14. Brown SL, Middleton MS, Berg WA, Soo MS, Pennello G (2000) Prevalence of rupture of silicone gel breast implants revealed on MR imaging in a population of women in Birmingham, Alabama. AJR Am J Roentgenol 175(4): 1057-1064

15. Silverman BG, Brown SL, Bright RA, Kaczmarek RG, ArrowsmithLowe JB, Kessler DA (1996) Reported complications of silicone gel breast implants: an epidemiologic review. Ann Intern Med 124(8): 744-756
16. Persellin ST, Vogler JB 3rd, Brazis PW, Moy OJ (1992) Detection of migratory silicone pseudotumor with use of magnetic resonance imaging. Mayo Clin Proc 67(9):891-895

17. Marcasciano M, Conversi A, Kaciulyte J, Dessy LA (2017) Prosthetic breast implant rupture: imaging-pictorial essay : full cooperation between surgeon and radiologist: "The Best of Both Worlds". Aesthet Plast Surg 41(6):1478-1480

18. Everson LI, Parantainen H, Detlie T, Stillman AE, Olson PN, Landis G, Foshager MC, Cunningham B, Griffiths HJ (1994) Diagnosis of breast implant rupture: imaging findings and relative efficacies of imaging techniques. AJR Am J Roentgenol 163(1): $57-60$

19. Herborn CU, Marincek B, Erfmann D, Meuli-Simmen C, Wedler V, Bode-Lesniewska B, Kubik-Huch RA (2002) Breast augmentation and reconstructive surgery: MR imaging of implant rupture and malignancy. Eur Radiol 12(9):2198-2206

20. Hirmand H, Hoffman LA, Smith JP (1994) Silicone migration to the pleural space associated with silicone-gel augmentation mammoplasty. Ann Plast Surg 32(6):645-647

21. Taupmann RE, Adler S (1993) Silicone pleural effusion due to iatrogenic breast implant rupture. South Med J 86(5):570-571

22. Rice DC, Agasthian T, Clay RP, Deschamps C (1995) Silicone thorax: a complication of tube thoracostomy in the presence of mammary implants. Ann Thorac Surg 60:14171419

23. Levine RL, Allen TC, Cartwright J, Cagle PT (2005) Silicone thorax due to a ruptured breast implant. Chest. 127:1854-1857

24. Tanaka T, Tao H, Hayashi T, Yoshiyama K, Furukawa M, Yoshida K, Okabe K (March 2015) Disseminated pleural siliconoma mimicking malignant pleural mesothelioma. Ann Thorac Surg 100: 2339-2340

25. Dragu A, Theegarten D, Bach SD, Polykandriotis E, Arkudas A, Kneser U, Horch RE, Ingianni G (2009) Intrapulmonary and cutaneous siliconomas after silent silicone breast implant failure. Breast J 15(5):496-499

26. Sykes JB, Rosella PA (2012) Intrathoracic migration of a silicone breast implant 5 months after video-Assisted thoracoscopic surgery. Comput Assist Tomogr 36:306-307

27. Lehoux JM, Tchantchaleishvili V, Jones CE (2013) Intrathoracic Migration of a silicone breast implant after video-assisted thoracoscopic surgery. Ann Thorac Surg 96: 326

28. Chen ZY, Wang ZG, Kuang RX, Wang BT, Su YP (2005) Implant found in thoracic cavity after breast augmentation. Plast Reconstr Surg 116(6):1826-1827

29. Kim H, Heo C, Beak R, Minn K (2009) Breast implant migration into pleural cavity. J Plast Reconstr Aesthet Surg 62:e89-e90

30. Fong TC, Hoffmann B (2011) Disappearance of a breast prosthesis during pilates. N Engl J Med 365(24):2305

31. Metha AM, Bard MP, van Straten A, van Beijeren I, Rijna H (2008) Intrathoracic migration of a breast prosthesis after thoracotomy. J Thorac Cardiovasc Surg 135(1):206-207 207.e1

32. Roussel LO, Koltz PF, Langstein HN (2015) Intrathoracic breast implant migration following video-Assisted thorascopic surgery. Plast Reconstr Surg 135(6):1075e-1076e

33. Colombo G, Ruvolo V, Stifanese R, Perillo M, Garlaschi A (2011) Prosthetic breast implant rupture: imaging-pictorial essay. Aesthet Plast Surg 35(5):891-900 
34. Swezey E, Shikhman R, Moufarrege R. Breast Implant Rupture. StatPearls [Internet]. Treasure Island (FL): StatPearls Publishing; 2020 Jan. 2020 Nov 1. PMID: 29083733 Bookshelf ID: NBK459308

35. Marcasciano M, Frattaroli J, Mori FLR, Lo Torto F, Fioramonti P, Cavalieri E, Kaciulyte J, Greco M, Casella D, Ribuffo D (2019) The new trend of pre-pectoral breast reconstruction: an objective evaluation of the quality of online information for patients undergoing breast reconstruction. Aesthet Plast Surg 43(3): 593-599

36. Casella D, Di Taranto G, Marcasciano M et al (2019) Subcutaneous expanders and synthetic mesh for breast reconstruction: long-term and patient-reported BREAST-Q outcomes of a single-center prospective study. J Plast Reconstr Aesthet Surg 72(5):805-812
37. Casella D, Di Taranto G, Marcasciano M et al (2019) Evaluation of prepectoral implant placement and complete coverage with tiloop bra mesh for breast reconstruction: a prospective study on long-term and patient-reported BREAST-Q outcomes. Plast Reconstr Surg 143(1):1e-9e

38. Marcasciano M, Kaciulyte J, Marcasciano F (2019) "No Drain, no gain": simultaneous seroma drainage and tissue expansion in prepectoral tissue expander-based breast reconstruction. Aesthet Plast Surg 43(4):1118-1119

Publisher's note Springer Nature remains neutral with regard to jurisdictional claims in published maps and institutional affiliations. 